\title{
Origins of Elastic Properties in Ordered Block Copolymer/Nanoparticle Composites
}

2004

Vol. 4, No. 12

2455-2459

\author{
R. B. Thompson, ${ }^{\star}$ K. Ø. Rasmussen, and T. Lookman \\ Theoretical Division, Los Alamos National Laboratory, \\ Los Alamos, New Mexico 87545
}

Received September 28, 2004; Revised Manuscript Received October 29, 2004

\begin{abstract}
We predict a diblock copolymer melt in the lamellar phase with added spherical nanoparticles that have an affinity for one block to have a lower tensile modulus than a pure diblock copolymer system. This weakening is due to the swelling of the lamellar domain by nanoparticles and the displacement of polymer by elastically inert fillers. Despite the overall decrease in the tensile modulus of a polydomain sample, the shear modulus for a single domain is unaffected by fillers.
\end{abstract}

Polymer nanocomposites are being extensively investigated because of the improvement in material properties that results from the addition of nanoscopic filler particles to the polymer matrix. ${ }^{1-4}$ In addition to their practical importance, such composites offer diverse scientific challenges, combining ideas from colloid science, polymer physics and chemistry, as well as material science. Polymer nanocomposites become even more interesting when the polymer matrix consists of a block copolymer, capable of self-assembling into a wide range of ordered nanoscaled structures; nanoparticles can then be sequestered in certain domains to form ordered nanocomposites. ${ }^{5-7}$ The simultaneous amphiphilic and colloidal selfassembly taking place in such ordered nanocomposites gives them complex structures ${ }^{8}$ and makes the structureproperty relationship particularly intriguing. Since there is little understanding of the mechanical properties that arise in ordered nanocomposites, we present in this theoretical work a first investigation of the origins of the elastic properties of an ordered nanocomposite with spherical nanofillers.

Buxton and Balazs ${ }^{9}$ have studied a phenomenological model of nanosphere-filled block copolymer systems in which a hybrid Cahn-Hilliard/Brownian dynamics simulation is used as input to a lattice spring model of the elastic moduli. Their approach provides a versatile and useful method of predicting properties but lacks polymeric detail in the elasticity portion of the simulation. Furthermore, they examine filled block copolymer systems in the solid state, where all morphological evolution is disregarded as the system is distorted.

\footnotetext{
* Corresponding author. E-mail: thompson@uwaterloo.ca. Present address: Department of Physics, University of Waterloo, 200 University Avenue West, Waterloo, Ontario, Canada N2L 3G1.
}

We examine the elastic properties of a melt state nanosphere filled block copolymer ordered nanocomposite using self-consistent field theory (SCFT). SCFT is a coarse-grained, first principles approach that has been successful in dealing with block copolymer structure..$^{10}$ In the framework of this theory, local monomer density profiles of different block copolymer chemical species are represented self-consistently using chemical potential fields. Both the densities and the fields are then used to determine the free energy for the system, and, if desired, the internal energies and entropies can be explicitly calculated. SCFT has been extended to deal with hard nanosphere/block copolymer nanocomposites by the incorporation of a density functional theory particle contribution. ${ }^{11,12}$ Further, Tyler and Morse have demonstrated that the linear elastic behavior of a melt block copolymer system, which is quasi-statically deformed, can be well characterized using SCFT. ${ }^{13}$ We have recently adapted this approach to an efficient real space, pseudo-spectral method ${ }^{14}$ and found an increasing elastic modulus in multiblock copolymer systems as a function of block number, in qualitative agreement with experiment. ${ }^{15}$ Here, we combine these two advances in order not only to predict the effect on the elastic properties of adding nanoparticles to a block copolymer melt but also to explain the physical origins of the observed effects.

We will study the prototypical system consisting of a symmetric AB diblock copolymer melt in the lamellar phase with added spherical nanoparticles that have an affinity for the A block of the copolymer. A schematic of this system is shown in Figure 1.

Consequently, a lamellar morphology with the particles sequestered in the A phase is being considered, and the 


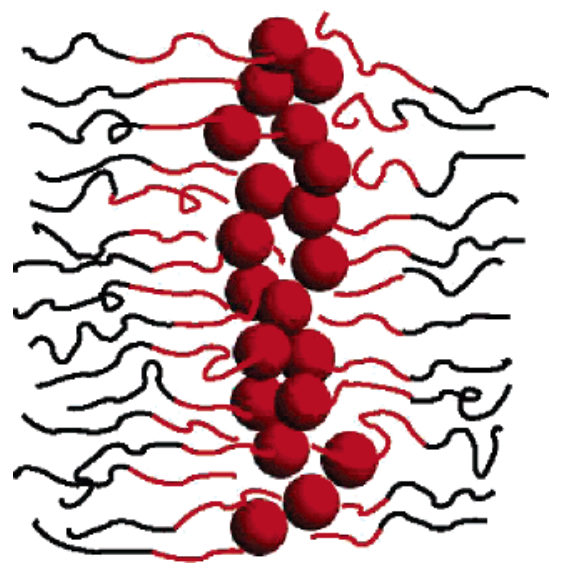

Figure 1. Illustration of a single domain of a block copolymer/ spherical nanoparticle-ordered composite material. The spheres are preferentially wetted by the A-block of the copolymer, shown in red. The black represents the B-blocks.

system's tetragonal symmetry is elastically characterized by just five independent nonzero components of the elastic modulus tensor. Additionally, the system is in a melt state so that deformations parallel to the lamellar structure have no effect on the free energy of the system. ${ }^{14}$ We are thus left with only two relevant moduli, $K_{33}$ and $K_{44}$. Therefore, we deform the system quasi-statically in two ways; it is subjected to an extension/compression, and to a simple shear. These deformations represent changes in the free energy caused by changes in the domain spacing, not changes in the moduli of the polymer melt or nanoparticle constituents; the mixture is taken to be incompressible ${ }^{16}$ and the particles are supposed to be nondeformable. ${ }^{17}$ These deformations allow us to determine the $K_{33}$ and $K_{44}$ components of the elastic modulus tensor, ${ }^{18}$ corresponding to extension/ compression and shear moduli, respectively. These components are found by taking the second derivative of the SCFT free energy with respect to the relative deformation. Greater detail on the methodology can be found in ref 14 .

We chose a system with a segregation of $\chi N=25$ between the $\mathrm{A}$ and $\mathrm{B}$ blocks, with the particles considered to be of the A species. $\chi$ is the Flory-Huggins monomer segregation parameter and $N$ is the degree of polymerization of the entire diblock. The particle radius was chosen to be $0.725 R_{\mathrm{g}}$, where $R_{\mathrm{g}}$ is the unperturbed radius of gyration of a diblock molecule. Finally, the particle-to-diblock volume ratio was $\sim 3.6$, and a $15 \%$ volume fraction of spherical fillers was added. The system was deformed in the two ways described above and compared with a neat diblock system similarly deformed. The morphology of the system before and after extensional deformation is shown in Figure 2.

The free energy as a function of the two deformations is shown in Figure 3; the pure diblock system is represented by solid curves and the $15 \%$ filled system by dashed curves. Figure $3 \mathrm{a}$ shows extensions and compressions whereas $3 \mathrm{~b}$ shows shear. The free energies in Figure 3 and hereafter are plotted as dimensionless free energies per volume, see for example eq 3. All curves in Figure 3 show parabolic shapes, indicating that linear elasticity theory is applicable. The curves were fitted with polynomials and the second deriva-

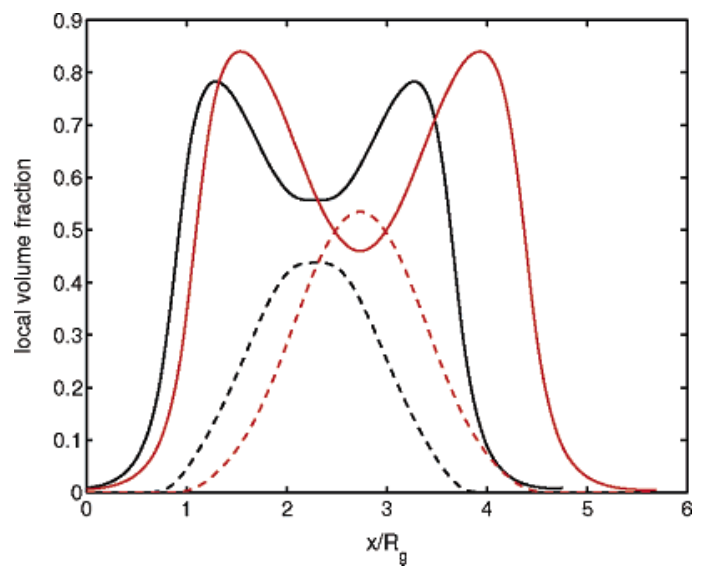

Figure 2. Density profiles for an ordered nanocomposite in the lamellar phase. The black lines are the A-block distribution (solid) and the particle distribution (dashed) before elongation. The corresponding red lines are the same distributions after stretching. The B block is not shown.
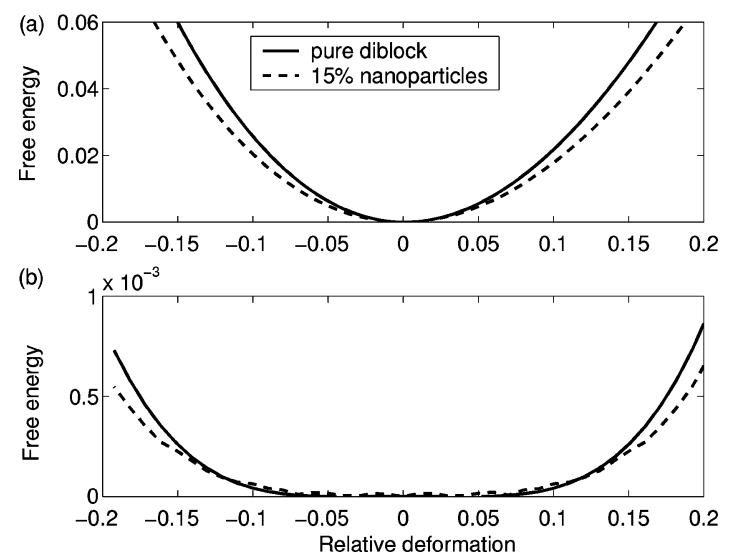

Figure 3. Total free energy versus relative distortion for extension/ compression (a) and shear (b) of a diblock system. The neat diblock free energy is plotted with the solid line whereas the $15 \%$ filled system is shown with dashed line. In (a), negative deformations are compressions while positive deformations are extensions. In all plots, energies have been zeroed around the equilibrium spacing, which is represented by a relative deformation $\epsilon=0$.

tives were used to find $K_{33}$ and $K_{44}$ for both the filled and unfilled systems according to the methodology of ref 14 . The $K_{33}$ and $K_{44}$ moduli in each case were used to find a tensile modulus by averaging over a polydomain sample according to the Hill prescription ${ }^{19}$ as described in ref 14 .

For the pure diblock system a dimensionless tensile modulus of $\sim 0.39$ was found while for the nanocomposite system, the modulus was $\sim 0.31$. Thus we predict that the addition of nanoparticles will weaken the material. For regular (homopolymer) nanocomposites, one expects the addition of nanofillers to strengthen the composite. ${ }^{2}$ The reduction in modulus found for our present system can be explained by decomposing the $K_{33}$ and $K_{44}$ moduli as shown in Tables 1 and 2.

These tables show total $K_{33}$ and $K_{44}$ moduli values, respectively, for $0 \%$ and $15 \%$ added fillers, as well as the difference between the filled and unfilled system moduli. The tables also give the contributions to the moduli of the internal energy $(U)$, translational entropy $\left(S_{\mathrm{T}}\right)$, and the A and 
Table 1. Components of the $K_{33}$ Elastic Modulus for $0 \%$ and $15 \%$ Added Nanoparticles ${ }^{a}$

\begin{tabular}{cccc}
\hline modulus & $0 \%$ & $15 \%$ & $\Delta$ modulus \\
\hline$K_{33}$ & 4.71 & 3.76 & -0.95 \\
$K_{33}^{U}$ & 2.80 & 1.95 & -0.85 \\
$K_{33}^{S_{\mathrm{T}}}$ & -0.58 & -0.60 & -0.02 \\
$K_{33}^{S_{\mathrm{A}}}$ & 1.23 & 0.49 & -0.74 \\
$K_{33}^{S_{\mathrm{B}}}$ & 1.25 & 1.58 & +0.33 \\
$K_{33}^{S_{\text {id }}}$ & 0.0 & 0.19 & +0.19 \\
$K_{33}^{S_{\text {st }}}$ & 0.0 & 0.14 & +0.14
\end{tabular}

${ }^{a}$ The change in the modulus between the pure diblock and the filled diblock system is recorded in the last column.

Table 2. Components of the $K_{44}$ Elastic Modulus for $0 \%$ and $15 \%$ Added Nanoparticles ${ }^{a}$

\begin{tabular}{cccc}
\hline modulus & $0 \%$ & $15 \%$ & $\Delta$ modulus \\
\hline$K_{44}$ & 0.00 & 0.00 & 0.00 \\
$K_{44}^{U}$ & 1.16 & 0.99 & -0.17 \\
$K_{44}^{S_{\mathrm{T}}}$ & -0.53 & -0.50 & +0.03 \\
$K_{44}^{S_{\mathrm{A}}}$ & -0.30 & -0.30 & 0.00 \\
$K_{44}^{S_{\mathrm{B}}}$ & -0.33 & -0.10 & +0.23 \\
$K_{44}^{S_{\text {id }}}$ & 0.00 & 0.00 & 0.00 \\
$K_{44}^{S_{\text {st }}}$ & 0.0 & -0.10 & -0.10
\end{tabular}

${ }^{a}$ The change in modulus between the pure diblock and the filled diblock system is recorded in the last column.

B conformational entropies of the diblocks $\left(S_{\mathrm{A}}\right.$ and $\left.S_{\mathrm{B}}\right)$. Also listed are the ideal gas $\left(S_{\mathrm{id}}\right)$ and steric $\left(S_{\mathrm{st}}\right)$ contributions of the filler particles. The moduli can be broken up into components like this because the second derivative of the free energy expression

$$
\mathscr{F}=\mathscr{U}-T\left(\mathcal{S}_{\mathrm{T}}+\mathcal{S}_{\mathrm{A}}+\mathcal{S}_{\mathrm{B}}+\mathcal{S}_{\mathrm{id}}+\mathcal{S}_{\mathrm{st}}\right)
$$

with respect to the relative deformation $\epsilon$

$$
\frac{\partial^{2} \mathscr{F}}{\partial \epsilon^{2}}=\frac{\partial^{2} \mathscr{U}}{\partial \epsilon^{2}}-T\left(\frac{\partial^{2} \mathcal{S}_{\mathrm{T}}}{\partial \epsilon^{2}}+\frac{\partial^{2} \mathcal{S}_{\mathrm{A}}}{\partial \epsilon^{2}}+\frac{\partial^{2} \mathcal{S}_{\mathrm{B}}}{\partial \epsilon^{2}}+\frac{\partial^{2} \mathcal{S}_{\mathrm{id}}}{\partial \epsilon^{2}}+\frac{\partial^{2} \mathcal{S}_{\mathrm{st}}}{\partial \epsilon^{2}}\right)
$$

is additive. In eqs 1 and 2, the caligraphic characters represent dimensionless energy per volume as defined, for example, in eq 3. Although the components of the free energy may not be parabolic like the total free energy, and may even have large first derivatives, it is the curvature of the components, which is to say, the second derivatives, that contribute to the total curvature, or moduli. For information on how these components are calculated, see ref 14. From Table 2 it is seen that the $K_{44}$ moduli for the filled and pure diblock systems were found to be very close to zero. Thus the main contribution to the tensile modulus arises from the extensional modulus $K_{33}$. Furthermore, the main drop in $K_{33}$ from the $0 \%$ to the $15 \%$ case is a result of the internal energy contribution and the A block conformational entropy contribution.

These two contributions can be examined separately. Upon extension/compression, SCFT shows that the lamellar interfacial width remains practically unchanged in both the filled
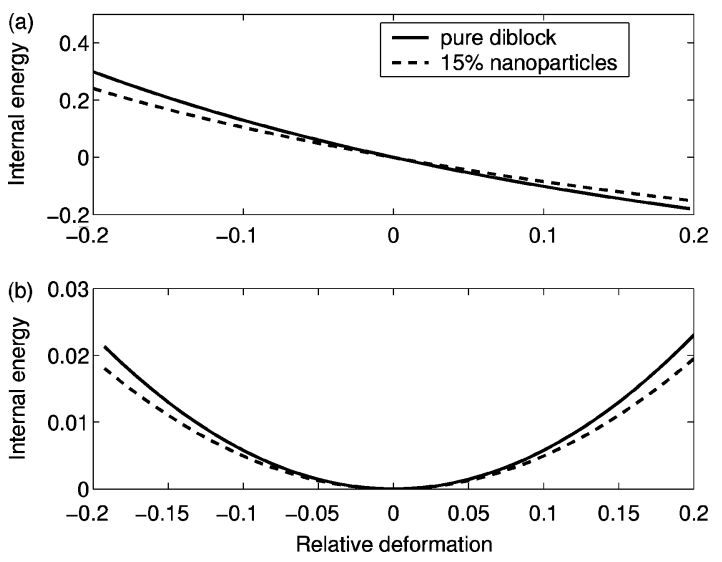

Figure 4. Internal energy contribution to the free energy versus relative distortion for extension/compression (a) and shear (b) of a diblock system. The neat diblock free energy is plotted with the solid line whereas the $15 \%$ filled system is shown with dashed line. In (a), negative deformations are compressions while positive deformations are extensions. In all plots, energies have been zeroed around the equilibrium spacing, which is represented by a relative deformation $\epsilon=0$.

and unfilled cases. Furthermore, the A and B domains are well segregated both before and after deformation, indicating that there are few $\mathrm{A}$ monomers in the $\mathrm{B}$ region and vice versa. Thus the absolute amount of energetically unfavorable $\mathrm{AB}$ contacts is the same before and after deformation, whereas the domain size changes. Adapting an expression of Matsen and Bates, ${ }^{20}$ the internal energy contribution to the free energy is

$$
\mathcal{U}=\frac{N U}{\rho_{0} V k_{\mathrm{B}} T} \propto \frac{1}{V} \int \mathrm{d} \mathbf{r}\left[\varphi_{\mathrm{A}}(\mathbf{r})+\varphi_{\mathrm{p}}(\mathbf{r})\right] \varphi_{\mathrm{B}}(\mathbf{r})
$$

where $T$ is the temperature, $k_{\mathrm{B}}$ is Boltzmann's constant, and $N$ is the degree of polymerization of an entire diblock based on a segment volume of $1 / \rho_{0} . \varphi_{\mathrm{A}}(\mathbf{r}), \varphi_{\mathrm{B}}(\mathbf{r})$, and $\varphi_{\mathrm{p}}(\mathbf{r})$ are the local volume fractions of $\mathrm{A}$ and $\mathrm{B}$ monomers and particles, respectively. The integral in eq 3 is constant under the conditions described above, so that the internal energy is inversely proportional to the volume $V$. The lamellar morphology is one-dimensional, so that the internal energy can be written as inversely proportional to the equilibrium domain size $d^{*}$.

$$
U=\frac{\alpha}{d^{*}}
$$

where $\alpha$ is a constant. A relative deformation $\epsilon$ that leaves the interfacial width and the bulk mixing unchanged changes eq 4 into

$$
u=\frac{\alpha}{d^{*}(1+\epsilon)}
$$

The SCFT internal energy for extensions/compressions is shown in Figure 4a. Both for pure diblock and for filled systems, the behavior reflected in eq 5 is observed. An 

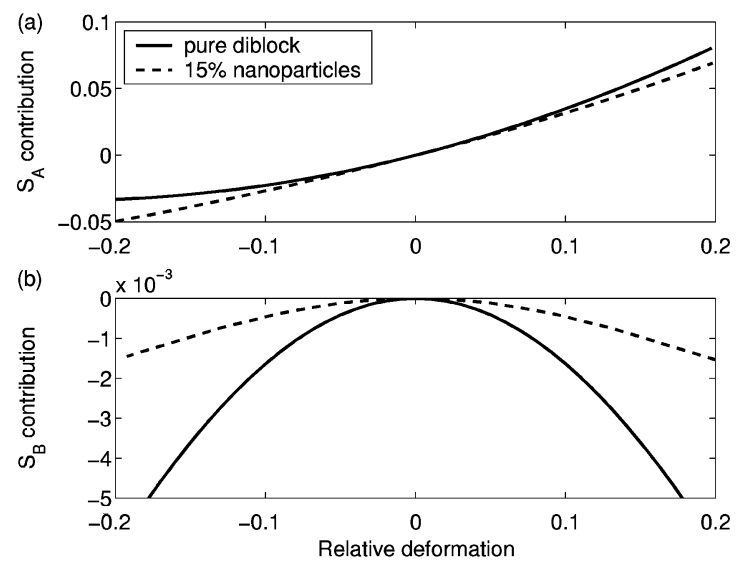

Figure 5. Conformational entropy contribution to the free energy versus distortion for extension/compression (a) and shear (b) of a diblock system. The neat diblock free energy is plotted with the solid lines whereas the $15 \%$ filled system is shown with dashed lines. In (a), negative deformations are compressions while positive deformations are extensions; the $S_{\mathrm{A}}$ contribution is shown in (a). The $S_{\mathrm{B}}$ contribution is shown in (b). In all plots, energies have been zeroed around the equilibrium spacing, which is represented by a relative deformation $\epsilon=0$.

estimate of the modulus $K_{33}^{U}$ can be found by taking the second derivative of eq 5 with respect to the relative deformation, which gives

$$
K_{33}^{U}=\left.\frac{d^{2} U}{d \epsilon^{2}}\right|_{\epsilon=0}=\frac{2 \alpha}{d^{*}}
$$

showing that the $U$ contribution to the $K_{33}$ modulus is also inversely proportional to the equilibrium spacing. SCFT shows that the addition of filler particles enlarges the equilibrium domain size of the nanocomposite compared to the pure diblock system. Equation 6 then indicates that the modulus will drop, as observed. In other words, the modulus is weakened partially because there is less interface per volume in the nanocomposite compared to the pure diblock.

The A block configurational entropy also contributes to the overall drop in modulus. The filler particles have no configurational entropy; in the filled system the $S_{\mathrm{A}}$ energy portion will rise (drop) under extension (compression) at a slower rate because there is a smaller fraction of chains to stretch (relax). This can be seen in Figure 5a. Thus, filler particles weaken the material because they displace polymers that have stretching energy, which could contribute positively to the elastic modulus. In other words, the modulus is weakened partially because there is less polymer and more elastically inert filler per volume.

As mentioned, the shear modulus $K_{44}$ makes a negligible contribution to the tensile modulus, but it is interesting to examine it nonetheless. Table 2 shows that both before and after the addition of nanoparticles the $K_{44}$ modulus is close to zero. Table 2 also shows that the components of $K_{44}$ do change in the presence of nanoparticles, but that these changes cancel out. A large increase in the B block conformational entropy contribution to the modulus is seen, but this is compensated for by a drop in the internal energy contribution and the particle steric term.
As in the extension/compression case, the interfacial profiles do not change significantly upon shearing of the sample. The consequence is that the internal energy again is inversely proportional to the volume of the system, as previously explained. The B block conformational entropy contribution to the free energy behaves similarly; from Matsen and Bates, ${ }^{20}$ the B block energy is

$$
-\mathcal{S}_{\mathrm{B}}=\frac{-N S_{\mathrm{B}}}{\rho_{0} V k_{\mathrm{B}}} \propto-\frac{1}{V} \int \mathrm{d} \mathbf{r}\left\{\rho_{\mathrm{J}}(\mathbf{r}) \ln q^{\dagger}(\mathbf{r}, f)+w_{\mathrm{B}}(\mathbf{r}) \varphi_{\mathrm{B}}(\mathbf{r})\right\}
$$

where $\rho_{\mathrm{J}}(\mathbf{r})$ is the distribution of diblock junction points, $w_{\mathrm{B}}(\mathbf{r})$ is the chemical potential field for the $\mathrm{B}$ monomer distribution, and $q^{\dagger}(\mathbf{r}, f)$ is an SCFT propagator. A detailed explanation of eq 7 can be found elsewhere. ${ }^{20,10}$ If we for the moment ignore the contribution of the integral in eq 7 , the $\mathrm{B}$ conformational contribution can be written as

$$
-\mathcal{S}_{\mathrm{B}}=-\frac{\beta}{V \sin \theta}
$$

where $\beta$ is a constant. $\mathrm{A} \sin \theta$ has been added to the denominator of eq 8 to account for a change in interfacial area upon shearing the sample. When the sample is sheared, the volume is constant but the $\mathrm{AB}$ interfacial area increases; $\theta$ is a measure of the amount of shear, with $\theta=\pi / 2$ representing a nonsheared system. See ref 14 for more explanations. The internal energy (eq 4) will be similarly affected. From ref 14, the shear angle is related to the relative distortion through $\epsilon=\cot \theta$. The internal energy and $\mathrm{B}$ conformational entropy are then

$$
\begin{gathered}
U=\frac{\alpha}{V} \sqrt{\epsilon^{2}+1} \\
-S_{\mathrm{B}}=-\frac{\beta}{V} \sqrt{\epsilon^{2}+1}
\end{gathered}
$$

Figures $4 \mathrm{~b}$ and $5 \mathrm{~b}$ show that the internal energy and B block contribution to the free energy obey the relationships of eqs 9 and 10, respectively, for both the filled and unfilled systems. The moduli for these are found through the second derivative with respect to $\epsilon$ and are

$$
\begin{gathered}
K_{44}^{U}=\frac{\alpha}{V} \\
K_{44}^{S_{\mathrm{B}}}=-\frac{\beta}{V}
\end{gathered}
$$

Both $K_{44}^{U}$ and $K_{44}^{S_{\mathrm{B}}}$ are inversely proportional to the equilibrium volume, with the important difference of a minus sign. Thus, as the equilibrium volume is enlarged upon the addition of fillers, the $S_{\mathrm{B}}$ contribution to the modulus becomes a smaller negative number, so the material would be stronger. This effect is countered by the $U$ contribution to the modulus, which becomes smaller for the larger equilibrium volume 
of the filled system. In other words, diblock molecules' entropies help shear the system: see Figure 5b. The larger domain size of the filled system means that there is less stretching energy per volume (see eq 10), the molecules are more relaxed to begin with at equilibrium, and therefore are less inclined to help deform the system, which makes the material stronger. This effect is countered since there is less interface per volume in the filled system, and the particles' excluded volumes interact more. The A block is not treated the same way, since with the fillers added the $S_{\mathrm{A}}$ contribution to the free energy cannot be written in a form such as eq 8 .

In summary, we have calculated the tensile modulus for a neat diblock copolymer system and for a diblock nanocomposite with $15 \%$ added nanospheres. Both systems were considered to be in the lamellar phase, and deformations were applied quasi-statically. The elastic modulus tensor components $K_{33}$ and $K_{44}$ were found and used to derive a tensile modulus for a polydomain sample. It was found that the addition of nanoparticles weakened the material. This was attributed to the larger lamellar domain size of the equilibrium filled system (it had less interface per volume) and to the displacement of polymer by the filler particles (there was less elastic polymer per volume). Although the shear modulus was negligible, it was acted on by similar mechanisms, with the components canceling each other. Given that ordered nanocomposites of the sort described here can now be realized, we believe our predictions should be amenable to experimental verification. It would be interesting to change the distribution of the particles in the block copolymer through changes of wetting properties, particle size, or volume fraction. Such changes in structure could significantly change the properties.

Acknowledgment. Work at the Los Alamos National Laboratory was performed under the auspices (contract W-7405-ENG-36) of the U.S. Department of Energy.

\section{References}

(1) Schmidt, G.; Malwitz, M. M. Curr. Opin. Colloid Interface Sci. 2003, 8,103 , and references therein.

(2) Balazs, A. C. Curr. Opin. Colloid Interface Sci. 2000, 4, 443, and references therein.

(3) Vaia, R. A.; Giannelis, E. P. MRS Bull. 2001, 26, 394, and references therein.

(4) Vaia, R. A., Krishnamoorti, R., Eds. Polymer Nanocomposites; American Chemical Society: Washington, DC, 2001

(5) Bockstaller, M.; Kolb, R.; Thomas, E. L. Adv. Mater. 2001, 13, 1783 .

(6) Bockstaller, M. R.; Lapetnikov, Y.; Margel, S.; Thomas, E. L. J. Am. Chem. Soc. 2003, 125, 5276.

(7) Jain, A.; Gutmann, J. S.; Garcia, C. B. W.; Zhang, Y.; Tate, M. W.; Gruner, S. M.; Wiesner, U. Macromolecules 2002, 35, 4862.

(8) Thompson, R. B.; Lee, J. Y.; Jasnow, D.; Balazs, A. C. Phys. Rev. E 2002, 66, 031801. Thompson, R. B.; Lee, J. Y.; Jasnow, D.; Balazs, A. C. Phys. Rev. Lett. 2002, 89, 155503. Thompson, R. B.; Lee, J. Y.; Jasnow, D.; Balazs, A. C. Faraday Discuss. 2003, 123, 121.

(9) Buxton, G. A.; Balazs, A. C. Phys. Rev. E 2003, 67, 031802.

(10) Matsen, M. W. J. Phys.: Condens. Mater. 2002, 14, R21.

(11) Thompson, R. B.; Ginzburg, V. V.; Matsen, M. W.; Balazs, A. C. Science 2001, 292, 2469. Thompson, R. B.; Ginzburg, V. V.; Matsen, M. W.; Balazs, A. C. Macromolecules 2002, 35, 1060.

(12) Balazs, A. C. Curr. Opin. Solid State Mater. 2003, 7, 27.

(13) Tyler, C. A.; Morse, D. C. Macromolecules 2003, 36, 3764.

(14) Thompson, R. B.; Rasmussen, K. Ø.; Lookman, T. J. Chem. Phys. 2004, 120, 3990.

(15) Spontak, R. J.; Smith, S. D. J. Polym. Sci., Part B: Polym. Phys. 2001, 39, 947.

(16) The incompressible system must have a Poisson ratio of 0.5 . The present work therefore addresses only elasticity arising from deformation of the periodic structure and not from the contituents themselves. It is possible to modify SCFT to account for compressibility, but this is beyond the scope of the present work.

(17) It would also be possible to allow the particles to be deformed as the mixture is distorted, but this would not correspond to the quasistatic melt state deformation that we are considering here. This would correspond instead to a system of softer nanoparticles and would be more appropriately studied without the addition of a density functional term.

(18) Landau, L. D.; Lifshitz, E. M. Theory of Elasticity; ButterworthHeinemann: Oxford, 1999.

(19) Schreiber, E.; Anderson, O. L.; Soga, N. Elastic Constants and Their Measurement; McGraw-Hill: Toronto, 1973.

(20) Matsen, M. W.; Bates, F. S. J. Chem. Phys. 1997, 106, 2436.

NL048407F 